Juan Tortosa, Mar.

Alumna de doctorado e investigadora FPI en la Facultad de Bellas Artes de la Universidad Politècnica de València, en el Departamento de Escultura dentro del grupo de investigación el Laboratorio de Creaciones Intermedia.

\title{
Fusión de disciplinas en los nuevos movimientos plásticos contemporáneos: joyería contemporánea y arte objetual como híbrido a través del cuerpo.
}

\section{Fusion of disciplines in the new contemporary artistic movements: contemporary jewellery and objectual art as a hybid trhough the body.}

\section{TIPO DE TRABAJO:}

Comunicación.

PALABRAS CLAVE:

Cuerpo, objeto, extensiones, arte objetual, joyería contemporánea.

KEY WORDS:

Body, object, extensions, object art, contemporary jewellery.

\section{RESUMEN.}

“¿Rebecca Horn sabía en 1972 que había hecho Joyería Contemporánea con Finguers Gloves?”

En las últimas décadas una parte de las Artes Aplicadas ha evolucionado desplazándose hacia el plano de las Artes Plásticas a través de nuevos movimientos contemporáneos. Así mismo, las Artes Plásticas se han acercado a reconsiderar la importancia del valor de un uso o aplicación. Como cualquier componente vivo, estas disciplinas evolucionan, se metamorfosean o mutan entre ellas.

En la Joyería Contemporánea, hay un campo que traspasa el plano de las Artes Aplicadas, zambulléndose en la experimentación y proyección de las Artes Plásticas. Joyas/objetos interaccionan con el cuerpo desafiando las leyes de la ergonomía y aplicación. Éstas establecen muchos paralelismos con otras dentro del Arte Objetual donde también se trabaja con el cuerpo como campo de investigación y experimentación, a través de la interacción entre individuo y objeto. Estamos ante obras que aparecen en el cuerpo como un conjunto performativo que reflexionan acerca del mismo. Objetos que nacieron de la experimentación tanto a nivel formal como conceptual, desprendiéndose de materiales estandarizados y del lujo para dar paso a algunas preguntas en torno a él.

Los códigos de la Joyería Contemporánea y del Arte Objetual, lo que ha hecho que evolucione de esta manera, cómo se acoge este híbrido, cuál es la línea que separa la obra de Rebecca Horn y Lauren Kalman, Ursula Guttmann, etc. son algunos de los puntos que se abarcan en esta investigación junto con un proyecto plástico, basado en el estudio del cuerpo y sus extensiones, y las diferentes maneras que tenemos de relacionarnos con él y su entorno.

\section{ABSTRACT.}

Did Rebecca Horn know in 1972 that she had made Contemporary Jewellery with Finguers Gloves?

In recent decades a part of Applied Arts have evolved to move towards the plane of the Visual Arts through new contemporary movements. Likewise, the Visual Arts have approached to reconsider the importance of the value of use or application. As any living component, these disciplines evolve, metamorphose or mutate among them. 
In contemporary jewellery, there is a field that crosses the plane of the Applied Arts, diving into experimentation and Visual Arts' projection. Jewellery/objects interact with the body, defying the laws of ergonomics and application. They established many parallels with others within the Object Art where we can work also with the body as a field of research and experimentation, through the interaction between individual and object. We are in front of works which appear in the body as a performance group that speaks about it. Objects that were born from experimentation and at the same time from a concept, they lose standardized materials and luxury to open a way for some questions about the body.

The Contemporary Jewellery and Object Art codes, which provoke this sort of evolving, how do we see this hybrid, which is the line that divides Rebecca Horn's work with Lauren Kalman's work, Ursula Guttmann's work, etc. They are some of the study items that involve this investigation together with a practical project based in the body study and its extensions, and the different ways that we have for interact with it and its environment.

\section{CONTENIDO.}

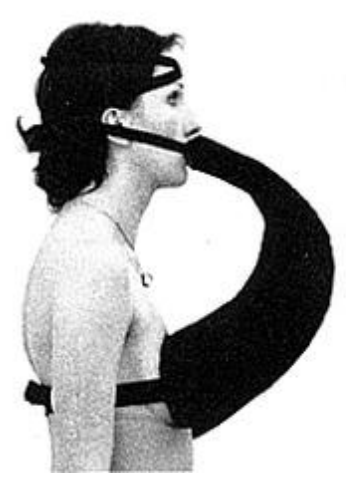

Todo está vivo, evoluciona, se metamorfosea o muta. Las Artes Plásticas no iban a ser menos, al igual que las Artes Aplicadas. Lo hemos podido observar estas últimas décadas a través de la pluralidad de sus obras, la expansión entre campos de ejecución y la variedad de recursos que se están utilizando.

Encontrar la línea que separa unas disciplinas de otras, es cada vez más difícil. Este suceso no es para nada negativo, si partimos de la base del conocimiento. Saber cuál es el campo de trabajo en el que estamos o del que partimos, cuál es la disciplina a la que te acoges y cuáles son sus parámetros, nos da las herramientas para poder realizar un proyecto sólido y que no sea ambiguo de manera inconsciente. El conocimiento brinda la oportunidad de ofrecer más variedad de recursos con rigor, de elegir aquello preestablecido y acotado o un híbrido entre varias disciplinas, de libertad. Esta es la clave para un proyecto más rico en recursos y contenidos.

En esta tesis se habla del híbrido que hay entre la Joyería Contemporánea de carácter más experimental y el Arte Objetual aplicado al cuerpo. Se están estudiando cuáles son las líneas que separan estas disciplinas pertenecientes a diferentes campos de trabajo y cuáles son las que las unen y la retroalimentación que hay entre las dos. Se está realizando un análisis para así entender mejor la evolución que se está experimentando, la cual, al igual que nosotros está viva y cambiante y puede adoptar una posición híbrida, metamorfosear o mutar. Son tiempos donde a pesar del carácter originario, las Artes Plásticas nos ofrecen aplicaciones y las Artes Aplicadas nos ofrecen contemplación.

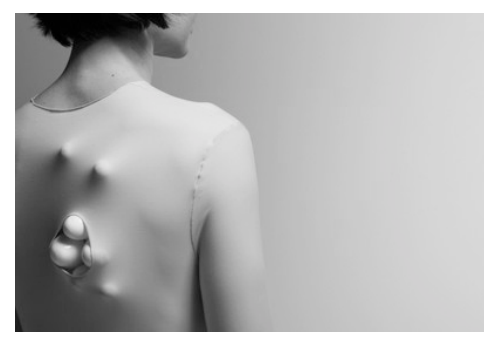

\section{OBJETO COMO DETONANTE EN LA REFLEXIÓN ARTÍSTICA.}

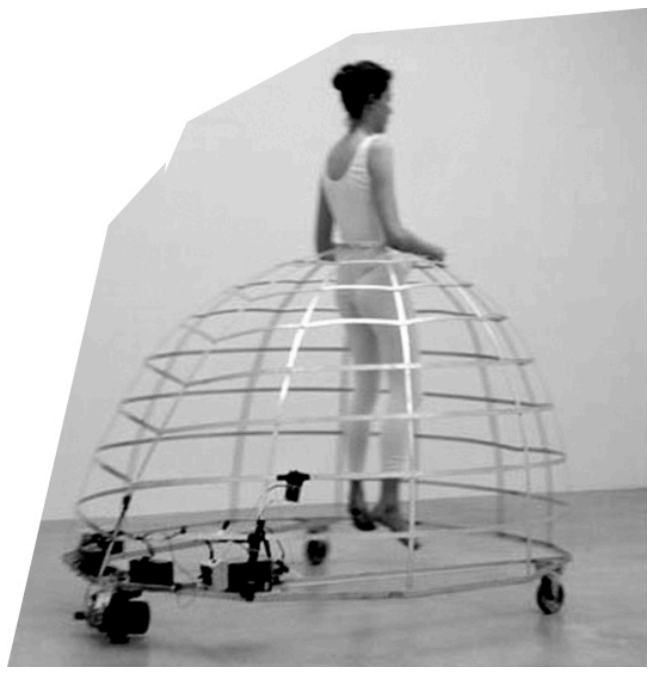

Cuerpo, objeto que nos limita y a la vez medio por el cual expandirnos. Como seres humanos, tenemos la necesidad de ser, vivir, sentir, expresarnos y relacionarnos. Día a día conformamos una manera de ser que nos permite desarrollarnos como personas y descubrir tanto el funcionamiento de la sociedad en la que estamos inmersos, como nuestro propio funcionamiento. El cuerpo como objeto es el medio por el cual descifrar los códigos y a la par conformar los nuestros para así comunicarnos.

A lo largo de nuestra vida y de su proceso de entendimiento, nos encontramos trabas y barreras en la sociedad o en nosotros mismos que nos impiden, a veces, llegar a ciertos estados o situaciones, los cuales por cualquier motivo (personal, reivindicativo, reflexivo, contemplativo, etc.) tenemos la necesidad de experimentar.

Así como el pintor puede pintar con su cuerpo, también lo hace a través de herramientas que extienden su capacidad de depositar la pintura o materia, de una manera específica en el lienzo para poder plasmar su fin estético en la obra. Estamos en constante convivencia con los objetos, comemos con cubiertos, 
vemos mejor con gafas y a falta de muela buena es una prótesis. Estos son algunos ejemplos que se podrían citar para describir la relación que tenemos con los objetos, objetos-extensiones, objetos-prótesis, etc.

Vivimos en una sociedad materialista. La gran mayoría de los objetos que tenemos en nuestro entorno son extensiones que se han fabricado para hacernos "más fácil" la existencia. El coche nos permite ir más rápido, el teléfono comunicarnos verbalmente de manera no presencial, etc. Está claro que nuestro cuerpo nos permite hacer prácticamente todo, lo que no podemos es alterar la manera, rapidez, duración, precisión, etc. con la que queremos hacer un determinado tipo de acción.

Como podemos observar hay una íntima relación entre los objetos y nuestro cuerpo, los objetos y nosotros. Por lo tanto no es de extrañar, que artistas se amarren a este tema para desarrollar su obra y veamos representados esos objetos a modo de extensiones, complementos o aportaciones en el cuerpo, para así realizar o experimentar una cierta acción o estado. Rebecca Horn, artista plástica, lo hizo con su obra "Finguers Gloves", ¿cómo llegar a los dos extremos de la pared a la vez? ¿Cómo cogeríamos los objetos si conseguimos extender nuestros brazos o manos? Unos dedos larguísimos colocados a modo de guante, nos hacen ver y a ella experimentar, la expansión del cuerpo para así llegar a aquello impensado, una superficie lejana a nuestro cuerpo la cual queremos tocar a la par con los dos brazos, uno en cada extremo, como niño que anda con la mano pegada al muro y va sintiendo la textura de éste o su sonoridad, pero esta vez, con los dos extremos, de punta a punta de la pared.
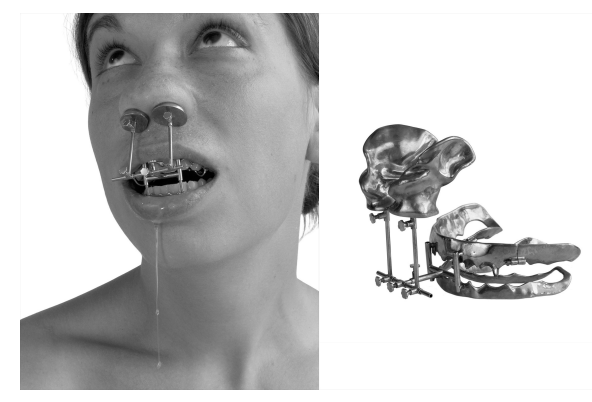

De 1972 pasamos al 2015 con Lauren Kalman, dentro de la joyería contemporánea. ¿Cómo habitar los espacios vacíos de nuestro cuerpo? La capacidad para sentir el espacio que ocupamos es mucho más accesible, ya que es nuestro propio cuerpo y por lo general tenemos la capacidad de sentir cómo está cada momento. Llegados a este punto, Kalman reflexiona en torno a los espacios desocupados muchas veces, aquellos que vienen dados por orificios de nuestro cuerpo (nariz, boca, oído, vagina, etc.). En su proyecto vemos como una serie de artilugios que se introducen en su cuerpo y ocupan el espacio vacío, lo hace físico. Pero vemos que va más allá, no encierra ese espacio físico en su cuerpo, sino que también lo expande. En muchas de las obras que componen "Device for filing a Void", vemos como el vacío cobra forma y podemos experimentar su fisicalidad, pero también a la vez, ver cómo se expande.

Hay muchos artistas y joyeros que trabajan de esta manera Lygia Clark, Ursula Guttmann, Peter Skubic, Anna Maria Staiano, Akiko Shinzado, etc. Pero hay algo que hace que este grupo de gente se separe en dos campos de trabajo aparentemente diferentes, y es que ciertos autores se engloban en el campo del Arte Objetual y otros en el de la Joyería Contemporánea de carácter experimental.

\section{PLURALIDAD}

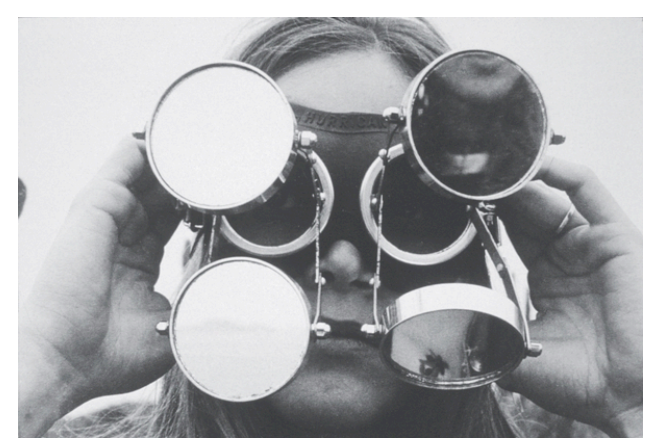

El arte contemporáneo oscila, entre otros motivos, por el sistema económico. En tiempos de crisis se planten salidas comerciales diferentes, o simplemente más amplias para que los artistas puedan sobrevivir. La fusión entre Artes Plásticas y Artes Aplicadas está más latente que nunca. No es de extrañar ver que un pintor lleva a cabo proyectos de diseño gráfico o un apasionado de la fotografía experimental fotografíe las piezas para un catálogo de productos de diseño. Es cuestión de poner bajo oficio esas cualidades potenciales que se tienen en otros campos que llevan implícita una necesidad para la sociedad. No solo se es creativo en la ejecución de la obra, sino también en las posibles salidas laborales y comerciales.

A la par, en los museos, galerías o salas expositivas podemos ver un amplio abanico de recursos expresivos a la hora de llevar a cabo un proyecto expositivo. Solo hay que ir a un museo de arte contemporáneo para ver que un mismo artista no se centra solo en una disciplina artística. Podemos encontrar fotografía, escultura, objeto, performance y video en la misma sala, una pluralidad de registro que hace de un todo más rico, que el lenguaje del arte se expanda a otros recursos, ya no solo en el lienzo, sino extendiéndose por las paredes, transportándose a objetos o reflejándose en una luz. El fin, en la mayoría de los casos, a parte de la maestría plástica, es llegar a una idea, un concepto, una visión, etc. la cual se entiende mejor en un determinado tipo de técnica o un estado físico.

Parte de esta diversidad es fruto de la sociedad en la que vivimos, ya sea por la situación económica, la pluralidad de recursos que adquirimos a medida que investigamos, como por la riqueza de información que está a nuestro alcance. Hoy por hoy, tenemos más información que nunca de forma libre y rápida. En internet podemos aprender a hacer desde una impresora láser a un pastel de chocolate. Tenemos acceso a un sinfín de conocimientos que pueden ayudarnos a enriquecer, no solo nuestro día a día, también nuestros recursos plásticos como artistas. No dependemos de alguien que nos enseñe (aunque siempre es mucho más eficaz si una persona nos sabe trasmitir bien los conceptos), podemos ser autosuficientes a la hora de encontrar recursos más exactos que se ciñan nuestro trabajo y su carácter. 
Al igual que vemos esta pluralidad en las Artes Plásticas, también se puede observar en la Joyería, concretamente en la Joyería Contemporánea de carácter más experimental. Los recursos que se utilizan se desprenden de los metales preciosos, van desde los polímeros más actuales que vienen por procesos de impresión de última tecnología, como son los sistemas de impresión a láser; hasta materiales como telas, escayolas, maderas, etc. Tanto materiales estándares como materiales de nuestro día a día, confluyen de forma paralela y enriquecen la manera de proyectar dentro de este ámbito y como resultado, la obra que se produce.

Pero no es solo la parte física la que está evolucionando, ahora también se mira más allá. El proceso de desarrollo de una joya hoy en día también abarca un proyecto, no estrictamente ligado a la

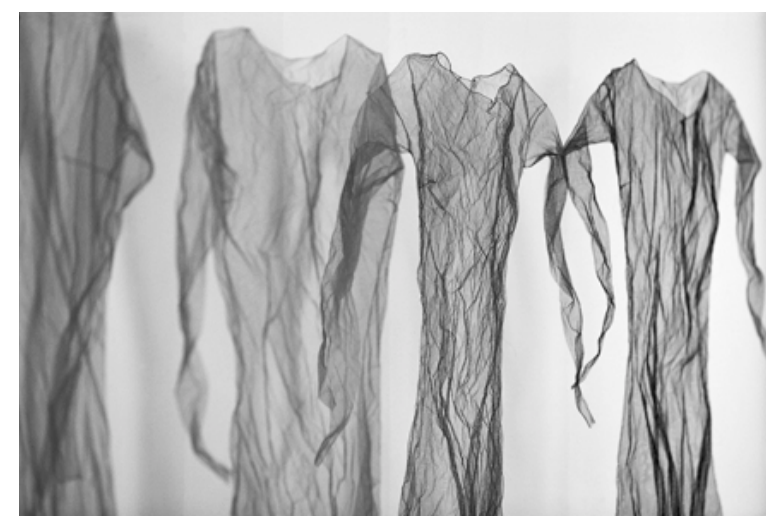
ergonomía sino que comparte premisas que oscilan entre el concepto, portabilidad o manera expositiva. Porque más allá de la estética, la joya ha sido un objeto que ha dado carácter visible a nuestra historia, estado económico, cívico, emocional, etc., ha fisicalizado un estado o una situación. Dado que ambos campos están intrínsecos en una joya, ¿por qué no trabajar también esta parte comunicativa de la joyería dentro de este ámbito y desprendernos de lo puramente ornamental?

$\underline{\text { HÍBRIDOS }}$

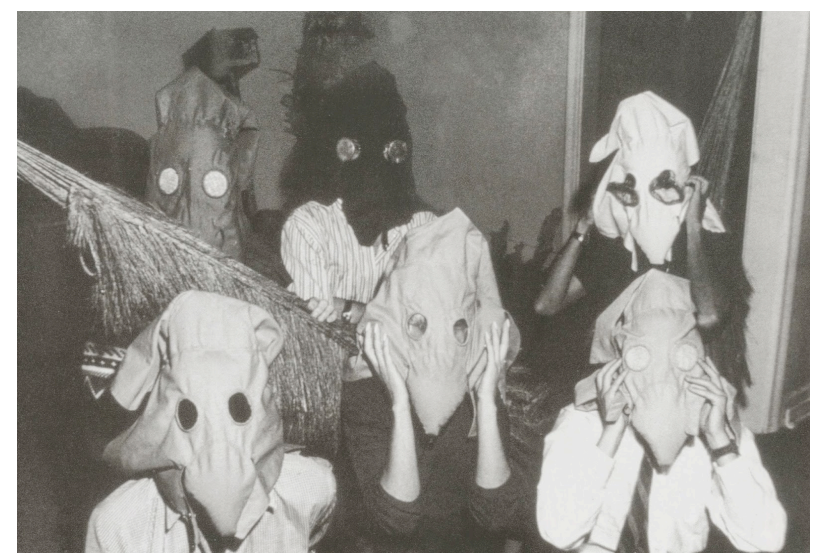

Poco a poco, hemos visto que dentro las Artes Plásticas, se ha adquirido un abanico de recursos más amplio, ya sea para enriquecer sus creaciones o para ampliar fronteras dentro del ámbito laboral, para documentar el proceso de un proyecto o para hacer un catálogo de diseño, a modo contemplativo o con un modo de aplicación. A la vez, en las Artes Aplicadas vemos que se está desarrollando cada vez más una parte reflexiva y experimental, la cual bebe de las Artes Plásticas para su desarrollo y que aunque no se ha tenido en cuenta durante muchos años, reside en su naturaleza.

Llegados a este punto, ¿dónde está la línea que separa las Artes Plásticas de las Artes Aplicadas? ¿Qué diferencia hay entre el Arte Objetual que se aplica en el cuerpo y la Joyería Contemporánea más experimental?

\section{IMÁGENES:}

1. Rebecca Horn, Cornucopia, Seance for Two Breasts, 1970.

2. Ursula Guttman, Tension, Organic Symbiosis, 2009.

3. Rebecca Horn, Performance.

4. Lauren Kalman, Device for Filling a Void, 2010.

5. Lygia Clark, Óculos,1968.

6. Caroline Broadhead, Ready to Tear, 1998.

7. Lygia Clark, Máscaras Sensoriais, 1967. 
Juan Tortosa, Mar

Fusión de disciplinas en los nuevos movimientos plásticos contemporáneos: joyería contemporánea y arte objetual como híbrido a través del cuerpo III CONGRESO INTERNACIONAL DE INVESTIGACIÓN EN ARTES VISUALES :: ANIAV 2017 :: GLOCAL [codificar, mediar, transformar, vivir] http://dx.doi.org/10.4995/ANIAV.2017.4913

\section{FUENTES REFERENCIALES.}

ANTONIO, J.A, 2009. El objeto y el aura. (Des)orden visual del arte moderno. Ed. AKAL / Arte Contemporáneo S.A.

BACHHUBER, L.; HOORMANN, A.; NIETO, J.; UNIVERSITAT DE VALÈNCIA, 2004. El objeto mismo: cuatro propuestas. CATÁLOGO. Valencia : Facultad de Bellas Artes de San Carlos, Universidad Politécnica de Valencia, D.L.

CABRAL ALMEIDA, A.M. 2014. La joyería contemporánea como arte. Un estudio filosófico. Tesis doctoral dirigida por VILAR G. Universidad Autónoma de Barcelona, Departamento de Filosofía.

CRUZ SÁNCHEZ, P. A.; HERNÁNDEZ, M. A. 2004. CARTOGRAFÍAS DEL CUERPO. La dimensión corporal en el arte contemporáneo. HD HOC, Serie Seminarios 4. Ed. Cendeac

VV.AA. , 2001. EXIT. №2: sobre la piel. Ed. Olivares \& Asociados, Madrid, 2001. ISSN 1577-2721

VV.AA. , 2001. EXIT. №42: el cuerpo como objeto. Ed. Olivares \& Asociados, Madrid, 2011. ISSN 1577-2721 